\title{
EQUATIONS WHICH CHARACTERIZE INNER PRODUCT SPACES
}

\author{
DAVID A. SENECHALLE
}

\begin{abstract}
It is shown that if $N$ is a normed linear space and there is a point $y$ of norm 1 such that an inequality of the type $a^{2}\|x\|^{2} \leqq \lim _{u \rightarrow 0} G\left(\left\{\left\|b_{i} u x+c_{i} y\right\|\right\}_{i=1}^{n}\right) \leqq b^{2}\|x\|^{2}$ holds for all $x$ in $N$ (where $0<a \leqq b$, the $c_{i}$ 's are nonzero and $G$ and $\|\cdot\|$ satisfy a certain twice-differentiability condition), then $N$ is isomorphic to an inner product space and $\inf \|T\| \cdot\left\|T^{-1}\right\| \leqq b / a$, where the infimum is taken over all linear homeomorphisms $T$ between $N$ and an inner product space. In the event that $a=b=1$, the inequality reduces to an equation which characterizes inner product spaces. An example shows that these results do not follow without the twice-differentiability condition on $G$.
\end{abstract}

S. O. Carlsson has proved [1] that a normed linear space $N$ is an inner product space if an equation of the type

$$
\sum_{i=0}^{n} a_{i}\left\|b_{i} x+c_{i} y\right\|^{2}=0
$$

(where the numbers $a_{i}$ are nonzero and the couples $\left(b_{i}, c_{i}\right)$ are pairwise linearly independent) holds for all $x$ and $y$ in $N$. An example of this type of equation is the Jordan-von Neumann condition for inner product spaces [2]:

$$
\|x+y\|^{2}+\|x-y\|^{2}=2\|x\|^{2}+2\|y\|^{2}
$$

for all $x$ and $y$ in $N$. A reformulation of Carlsson's condition is the following:

$$
\|x\|^{2}=\sum_{i=1}^{n} a_{i}\left\|b_{i} x+c_{i} y\right\|^{2}
$$

(where the coefficients are different from those in (1) and the numbers $c_{i}$ are nonzero) for all $x$ and $y$ in $N$. Now, if we define a function $G_{0}$ by $G_{0}\left(t_{1}, t_{2}, \cdots, t_{n}\right)=\sum_{i=1}^{n} a_{i} t_{i}^{2}$, then equation (3) says that, for all $x$ and $y$ in $N$,

$$
\|x\|^{2}=G_{0}\left(\left\{\left\|b_{i} x+c_{i} y\right\|\right\}_{i=1}^{n}\right) .
$$

Received by the editors October 16, 1972.

AMS (MOS) subject classifications (1970). Primary 46C10; Secondary 39A25.

(c) American Mathematical Society 1973 
In this paper Carlsson's condition is improved upon in several ways simultaneously: the specific function $G_{0}$ is replaced by a function $G$ which only need be twice-differentiable at a certain point, the requirement that (4) hold for all $y$ is replaced by the condition that it hold for a single $y$ (on the unit sphere for convenience) where the norm is twicedifferentiable, the requirement that (4) hold for all $x$ is replaced by a weaker limit condition, and the equality in (4) is replaced by inequalities which give information on the nearness of $N$ to an inner product space in case $N$ is isomorphic but not isometric to an inner product space. The main result of this paper is: If there is a point $y$ on the unit sphere and some function $G$ such that an inequality of the type

$$
a^{2}\|x\|^{2} \leqq \lim _{u \rightarrow 0} \frac{G\left(\left\{\left\|b_{i} u x+c_{i} y\right\|\right\}_{i=1}^{n}\right)}{u^{2}} \leqq b^{2}\|x\|^{2}
$$

holds for all $x$ in $N$ (where $0<a \leqq b$, the numbers $c_{i}$ are nonzero, and $G$ and the norm satisfy a certain twice-differentiability condition), then $N$ is isomorphic to an inner product space. Furthermore, letting $K(N)=$ $\inf \left\{\|T\| \cdot\left\|T^{-1}\right\|: T\right.$ is an isomorphism from $N$ onto an inner product space $\}$, it follows that $K(N) \leqq b / a$. (This means that an inner product $((\cdot, \cdot))$ exists such that $a^{2}\|x\|^{2} \leqq((x, x)) \leqq b^{2}\|x\|^{2}$ for all $x$ in $N$.) An example shows that these results do not follow without the twice-differentiability condition on $G$. The twice-differentiability condition on the norm can be removed if we require that (5) hold for all $x$ and $y$ in $N$.

Say that a function $G$ from a subset $A$ of a normed linear space $N_{1}$ into a normed linear space $N_{2}$ is twice-differentiable at the point $x$ of $A$ if $x$ is a limit point of $A$ and their exist a bounded linear mapping $T_{1}$ from $N_{1}$ into $N_{2}$ and a bounded bilinear mapping $T_{2}$ from $N_{1} \times N_{1}$ into $N_{2}$ such that

$$
\lim _{\varepsilon \rightarrow 0, x+\varepsilon \in A} \frac{G(x+\varepsilon)-G(x)-T_{1}(\varepsilon)-\frac{1}{2} T_{2}(\varepsilon, \varepsilon)}{\|\varepsilon\|^{2}}=0 .
$$

This definition does not require that $G$ be differentiable at points of $A$ other than $x$. The necessary lemmas on this subject are stated at the end.

Throughout this paper, $N$ denotes a normed linear space. An $n$-tuple $\left(t_{1}, t_{2}, \cdots, t_{n}\right)$ is denoted $\left\{t_{i}\right\}$. If $t_{0}, t_{1}, \cdots, t_{n}$ are numbers or points, $\left\{t_{i}\right\}$ denotes $\left(t_{1}, t_{2}, \cdots, t_{n}\right)$, not $\left(t_{0}, t_{1}, \cdots, t_{n}\right)$.

THEOREM 1. Suppose that there exist a function $G$ from $N$ into $\boldsymbol{R}$ which is twice-differentiable at 0 and numbers $a$ and $b, 0<a \leqq b$, such that for all $x$ in $N, a^{2}\|x\|^{2} \leqq \lim _{u \rightarrow 0} G(u x) / u^{2} \leqq b^{2}\|x\|^{2}$. Then $K(N) \leqq b / a$.

Proof. Let $T_{1}$ and $T_{2}$ be derivatives of $G$ at 0 , as in the above definition. 
Then

$$
\lim _{x \rightarrow 0} \frac{G(x)-G(0)-T_{1}(x)-\frac{1}{2} T_{2}(x, x)}{\|x\|^{2}}=0 .
$$

This implies that $G$ is continuous at 0 , and since $\lim _{u \rightarrow 0} G(u x) / u^{2}$ exists, $G(0)=0$. For all $x$ in $N, 0=\lim _{u \rightarrow 0}\left(G(u x)-u T_{1}(x)-\frac{1}{2} u^{2} T_{2}(x, x)\right) / u^{2}=$ $\left(\lim _{u \rightarrow 0} G(u x) / u^{2}\right)-\frac{1}{2} T_{2}(x, x)-\left(\lim _{u \rightarrow 0} T_{1}(x) / u\right)$. Therefore, $T_{1}=0$ and $\lim _{u \rightarrow 0} G(u x) / u^{2}=\frac{1}{2} T_{2}(x, x)$. For all $x$ and $y$ in $N$, define $((x, y))=$ $\left(T_{2}(x, y)+T_{2}(y, x)\right) / 4$. Then $((\cdot, \cdot))$ is an inner product for $N$ satisfying $a^{2}\|x\|^{2} \leqq((x, x)) \leqq b^{2}\|x\|^{2}$ for all $x$ in $N$. This implies $K(N) \leqq b / a$.

COROLlARY 1.1. If the square of the norm is twice-differentiable at 0 , then $N$ is an inner product space.

THEOREM 2. Suppose that there exist a point $y$ of norm 1 where $\|\cdot\|$ is twice-differentiable, numbers $b_{i}$ and $c_{i}, c_{i} \neq 0, i=1, \cdots, n$, a function $G$ from a subset of $\boldsymbol{R}^{n}$ into $\boldsymbol{R}$ twice-differentiable at $\left\{\left|c_{i}\right|\right\}$, and numbers $a$ and $b$, $0<a \leqq b$, such that for every $x$ in $N, a^{2}\|x\|^{2} \leqq \lim _{u \rightarrow 0} G\left(\left\{\left\|b_{i} u x+c_{i} y\right\|\right\}\right) / u^{2}$ $\leqq b^{2}\|x\|^{2}$. Then $K(N) \leqq b / a$.

Proof. Let $\mathscr{N}=N_{1} \times N_{2} \times \cdots \times N_{n}$, where each $N_{i}=N$. Then define functions $E: \boldsymbol{R} \rightarrow \mathscr{N}$ and $F: \mathscr{N} \rightarrow \boldsymbol{R}^{n}$ by $E(u)=\left\{b_{i} u x+c_{i} y\right\}$ and $F\left(\left\{p_{i}\right\}\right)=$ $\left\{\left\|p_{i}\right\|\right\}$. Then $E$ is twice-differentiable at $0, F$ is twice-differentiable at $E(0)$, and $G$ is twice-differentiable at $F \circ E(0)$. By two applications of Lemma 1 on twice-differentiable functions, $G \circ F \circ E$ is twice-differentiable at 0 . By the hypothesis $a^{2}\|x\|^{2} \leqq \lim _{u \rightarrow 0} G \circ F \circ E(u x) / u^{2} \leqq b^{2}\|x\|^{2}$ for all $x$ in $N$. Theorem 1 then asserts that $K(N) \leqq b / a$.

One immediate consequence of Theorem 2 is the following improvement on the Jordan-von Neumann condition:

COROLlary 2.1. If there exist a point y of norm 1 in $N$ where $\|\cdot\|$ is twice-differentiable and numbers $a$ and $b, 0<a \leqq b$, such that for all $x$ in $N$,

$$
a^{2}\|x\|^{2} \leqq \lim _{u \rightarrow 0} \frac{\|u x+y\|^{2}+\|u x-y\|^{2}-2\|y\|^{2}}{2 u^{2}} \leqq b^{2}\|x\|^{2},
$$

then $K(N) \leqq b / a$.

Proof. It is just a matter of checking that the function $G\left(t_{1}, t_{2}, t_{3}\right)=$ $\left(t_{1}^{2}+t_{2}^{2}-2 t_{3}^{2}\right) / 2$ is twice-differentiable at $(1,1,1)$.

If $N$ is 2-dimensional, then Lemma 2 on twice-differentiable functions may be used to eliminate from the hypothesis of Corollary 2.1 the condition that there exist a point $y$ of norm 1 where $\|\cdot\|$ is twice-differentiable.

COROllary 2.2. Suppose that there exist a point $y$ of norm 1 where $\|\cdot\|$ is twice-differentiable, pairwise linearly independent points in the plane 
$\left(b_{i}, c_{i}\right), i=0,1, \cdots, n$, a function $G$ twice-differentiable at

$$
\left\{\left|b_{0} c_{i}-c_{0} b_{i}\right| /\left(b_{0}^{2}+c_{0}^{2}\right)^{1 / 2}\right\},
$$

and numbers $a$ and $b, 0<a \leqq b$, such that for all $p$ and $q$ in $N, a^{2}\left\|b_{0} p+c_{0} q\right\|^{2} \leqq$ $G\left(\left\{\left\|b_{i} p+c_{i} q\right\|\right\}\right) \leqq b^{2}\left\|b_{0} p+c_{0} q\right\|^{2}$. Then $K(N) \leqq b / a$.

Proof. Define $b_{i}^{\prime}=\left(b_{0} b_{i}+c_{0} c_{i}\right) /\left(b_{0}^{2}+c_{0}^{2}\right)^{1 / 2}, c_{i}^{\prime}=\left(b_{0} c_{i}-c_{0} b_{i}\right) /\left(b_{0}^{2}+c_{0}^{2}\right)^{1 / 2}$, $i=0,1, \cdots, n$. Then the $c_{i}^{\prime}$ are nonzero and $\left(b_{0}^{\prime}, c_{0}^{\prime}\right)=(1,0)$. Suppose $x$ is in $N$. Let $p=\left(b_{0} x-c_{0} y\right) /\left(b_{0}^{2}+c_{0}^{2}\right)^{1 / 2}$ and $q=\left(c_{0} x+b_{0} y\right) /\left(b_{0}^{2}+c_{0}^{2}\right)^{1 / 2}$. Then

$$
\begin{aligned}
a^{2}\|x\|^{2} & =a^{2}\left\|b_{0} p+c_{0} q\right\|^{2} \leqq G\left(\left\{\left\|b_{i} p+c_{i} q\right\|\right\}\right) \\
& =G\left(\left\{\left\|b_{i}^{\prime} x+c_{i}^{\prime} y\right\|\right\}\right) \leqq b^{2}\left\|b_{0} p+c_{0} q\right\|^{2}=b^{2}\|x\|^{2} .
\end{aligned}
$$

By Theorem 2, $K(N) \leqq b / a$.

Lemma 2 on twice-differentiable functions may be used to omit from the hypothesis of the above theorem the condition on the existence of the point $y$ if the conclusion is altered to read " $K\left(N^{\prime}\right) \leqq b / a$ for every two-dimensional subspace $N^{\prime}$ of $N$." However, this does not insure that $K(N) \leqq b / a$.

To get Carlsson's theorem from Corollary 2.2, define

$$
G\left(t_{1}, t_{2}, \cdots, t_{n}\right)=\frac{-1}{a_{0}} \sum_{i=1}^{n} a_{i} t_{i}^{2}
$$

and note that $G$ is twice-differentiable everywhere. Then, using Lemma 2 on twice-differentiable functions, we have that every 2-dimensional subspace of $N$ is an inner product space, and, hence, that $N$ is an inner product space.

If $n=3$, then the condition that $G$ be twice-differentiable may be omitted from the hypothesis of Theorem 2, as this author has shown in [3, Theorem 6]. One is tempted to guess that for other values of $n$ that condition is also unnecessary. The following example disproves that conjecture.

In the plane let $A$ be the arc $\left\{(a, b): b \geqq 0, a^{2}+\left(b+\frac{1}{2}\right)^{2}=1\right\}$ of the circle with radius 1 and center $\left(0,-\frac{i}{2}\right)$. Let $S$ be the unit sphere $A \cup(-A)$ and let $\|\cdot\|$ be the corresponding norm (the Minkowski functional for $S$ ). If $S^{\prime}$ is any linear image of $S$ distinct from $S$, then $S$ and $S^{\prime}$ have no more than 3 pairwise linearly independent points in common. Let $\left(a_{i}, b_{i}\right)$, $i=0,1,2,3,4$, be five pairwise linearly independent points. We show that there is a function $G$ such that, for every two points $x$ and $y$,

$$
G\left(\left\{\left\|a_{i} x+b_{i} y\right\|\right\}\right)=\left\|a_{0} x+b_{0} y\right\|^{2} .
$$

Let $x$ and $y$ be points in the plane. By the strict convexity of $S, x$ and $y$ are linearly dependent if and only if there exist numbers $c$ and $d$ such that 
$\left\|a_{i} x+b_{i} y\right\|=\left|a_{i} c+b_{i} d\right|, i=1,2,3,4$, and in this case, define

$$
G\left(\left\{\left\|a_{i} x+b_{i} y\right\|\right\}\right)=\left(a_{0} c+b_{0} d\right)^{2},
$$

which is $\left\|a_{0} x+b_{0} y\right\|^{2}$. Suppose $x$ and $y$ are linearly independent. The four points $\left(a_{i}, b_{i}\right) /\left\|a_{i} x+b_{i} y\right\|, i=1,2,3,4$, belong to $T(S)$, where $T$ is the linear mapping such that $T(x)=(1,0)$ and $T(y)=(0,1)$. There exists no other linear image of $S$ containing these points. Let $k$ denote the positive number such that $\left(a_{0}, b_{0}\right) / k \in T(S)$. Then

$$
1=\left\|T^{-1}\left(\left(a_{0}, b_{0}\right) / k\right)\right\|=\left\|a_{0} x+b_{0} y\right\| / k,
$$

so $k=\left\|a_{0} x+b_{0} y\right\|$. Thus for $x$ and $y$ linearly independent, the rule is $G\left(\left\{\left\|a_{i} x+b_{i} y\right\|\right\}\right)=k^{2}$, where $k$ is the positive number such that $\left(a_{0}, b_{0}\right) / k$ belongs to the unique linear image of $S$ which contains $\left(a_{i}, b_{i}\right) /\left\|a_{i} x+b_{i} y\right\|$, $i=1,2,3,4$. Although such a function $G$ exists, $N$ is not an inner product space.

We have used the following two lemmas on twice-differentiable functions. The proof of Lemma 1 is omitted since it is quite similar to the proof of the ordinary composition theorem ("chain rule") in differential calculus.

LEMma 1. Suppose that $F$ is a function from a subset $A$ of the normed linear space $N_{1}$ into the normed linear space $N_{2}, x$ is a point of $A$ where $F$ is twice-differentiable, $G$ is a function from a subset of $N_{2}$ containing $F(x)$ into a normed linear space $N_{3}, G$ is twice-differentiable at $F(x)$, and $x$ is a member and limit point of $\operatorname{dom} F \circ G$. Let $S_{1}$ and $S_{2}\left(S_{1}\right.$ linear and $S_{2}$ bilinear) be functions satisfying the definition of twice-differentiability for $F$ at $x$, and let $T_{1}$ and $T_{2}$ be functions satisfying the definition for $G$ at $F(x)$. Then $G \circ F$ is twice-differentiable at $x$ and has derivatives $U_{1}=T_{1} \circ S_{1}$ and $U_{2}=T_{1} \circ S_{2}+T_{2} \circ\left(S_{1}, S_{1}\right)$.

LEMMA 2. Every norm defined on the plane is twice-differentiable almost everywhere.

Proof. Suppose $\|\cdot\|$ is a norm defined on the plane. Let $x$ and $y$ be two linearly independent points of the unit sphere, and let $r$ be the positive function such that $\|r(\theta) \cos (\theta) x+r(\theta) \sin (\theta) y\|=1$ for all $\theta$. Then $r$ is leftdifferentiable and for every $\theta,-\arctan \left(r_{-}^{\prime}(\theta) / r(\theta)\right)+\theta+\pi / 2$ gives the direction of the left-hand tangent to the unit sphere at $r(\theta) \cos (\theta) x+r(\theta) \sin (\theta) y$, so the function $-\arctan \left(r_{-}^{\prime}(\theta) / r(\theta)\right)+\theta+\pi / 2$ is nondecreasing and differentiable almost everywhere. It turns out that $r$ is twice-differentiable wherever $r_{-}^{\prime}$ is differentiable.

Suppose $r_{-}^{\prime}$ is differentiable at $\theta$. Then $r_{-}^{\prime}$ is continuous at $\theta$ and $r$ is differentiable at $\theta$. Let $T_{1}$ be the linear function and $T_{2}$ the bilinear function defined by $T_{1}(\varepsilon)=\varepsilon r^{\prime}(\theta)$ and $T_{2}(\varepsilon, \varepsilon)=\varepsilon^{2}\left(r_{-}^{\prime}\right)^{\prime}(\theta)$. For every $\varepsilon$, let 
$h(\varepsilon)=r(\theta+\varepsilon)-r(\theta)-T_{1}(\varepsilon)$. Then $h(0)=h^{\prime}(0)=0, h$ is left-differentiable, and $h_{-}^{\prime}$ is differentiable at 0 . It is a theorem that, under these conditions, $\lim _{\varepsilon \rightarrow 0} h(\varepsilon) / \varepsilon^{2}=\left(h_{-}^{\prime}\right)^{\prime}(0) / 2$. This implies that

$$
\lim _{\varepsilon \rightarrow 0} \frac{r(\theta+\varepsilon)-r(\theta)-T_{1}(\varepsilon)-\frac{1}{2} T_{2}(\varepsilon, \varepsilon)}{|\varepsilon|^{2}}=0,
$$

so $r$ is twice-differentiable almost everywhere.

The function $f$ from the plane into $\boldsymbol{R}$ defined by

$$
f(k \cos (\theta) x+k \sin (\theta) y)=\theta
$$

if $k>0$ and $\theta \in[0,2 \pi)$ is twice-differentiable except on the ray $\{k x: k \geqq 0\}$, and, hence, $r \circ f$ and $(r \circ f)^{-1}$ and the function $\|p\|=|p| /(r \circ f(p))$ are twice-differentiable almost everywhere.

\section{REFERENCES}

1. S. O. Carlsson, Orthogonality in normed linear spaces, Ark. Mat. 4 (1962), 297-318. MR 25 \#5364.

2. P. Jordan and J. von Neumann, On inner products in linear metric spaces, Ann. of Math. 2 (1935), 719-732.

3. D. A. Senechalle, Euclidean and non-Euclidean norms in a plane, Illinois J. Math. 15 (1971), 281-289. MR 43 \#6709.

Department of Mathematics, State University of New York, College at New Paltz, New Paltz, New York 12561 